\title{
Changes in blood glucose of elderly patients with gastric cancer combined with type 2 diabetes mellitus after radical operation and the effect of mediation adjustment for blood glucose on the recovery of gastric cancer
}

\author{
YUN ZHOU $^{1}$, SHIXIONG LIU ${ }^{1}$, JING WANG $^{1}$, XIANG YAN $^{1}$ and LEI ZHANG $^{2}$ \\ Departments of ${ }^{1}$ Gerontology, ${ }^{2}$ General Surgery, The First Hospital of \\ Lanzhou University, Lanzhou, Gansu 730000, P.R. China
}

Received September 26, 2017; Accepted June 28, 2018

DOI: 10.3892/ol.2018.9197

\begin{abstract}
This study aimed to investigate the changes in blood glucose of elderly patients with gastric cancer combined with type 2 diabetes mellitus (T2DM) after radical operation. Forty-six patients in DM group and 30 patients in non-DM group underwent radical surgery between January, 2007 and January, 2017 in The First Hospital of Lanzhou University, and the clinical data were retrospectively analyzed. Statistical analysis was conducted to explore the effect of radical operation on blood glucose control of the two groups of patients. In 46 patients with gastric cancer combined with T2DM, 24 patients received postoperative insulin intervention, and the remaining 22 patients did not receive insulin intervention. This latter set of patients comprised the non-insulin treatment group. Blood glucose control conditions, inflammatory markers, tumor markers and their effects on the prognosis were compared between the two groups. Level of postoperative blood glucose of the DM group was significantly higher than that of the non-DM group $(\mathrm{P}<0.05)$, while the level of blood glucose in the insulin treatment group was significantly lower than that in the control group $(\mathrm{P}<0.05)$. Levels of related tumor markers and inflammatory factors of insulin treatment group were significantly higher than those of the control group $(\mathrm{P}<0.05)$. The 5-year survival rate of insulin treatment group was significantly higher than that of the control group $(\mathrm{P}<0.05)$. Our results showed that the level of blood glucose was significantly increased in elderly patients with gastric cancer combined with T2DM after radical operation, and levels of blood glucose, tumor markers and inflammatory factors in elderly gastric cancer patients with
\end{abstract}

Correspondence to: Dr Shixiong Liu, Department of Gerontology, The First Hospital of Lanzhou University, 1 Dongang Xi Road, Chengguan, Lanzhou, Gansu 730000, P.R. China

E-mail: shixiong_liu02@163.com

Key words: gastric cancer, type 2 diabetes mellitus, postoperative blood glucose, prognosis
T2DM without insulin were significantly increased after radical operation, and long-term prognosis is poor.

\section{Introduction}

Gastric cancer is a relatively common gastrointestinal cancer. Relevant data have shown that the incidence of gastric cancer is on the increase, and gastric cancer tends to occur in the aging population. Gastric cancer is the third most common cause of cancer-related deaths worldwide (1). The main clinical manifestations of type 2 diabetes mellitus (T2DM) include insulin resistance and progressive decline of islet function, and it is commonly associated with systemic lipid metabolism (2). With an aging population that continues to grow, number of elderly patients with gastric cancer combined with T2DM is progressively increasing.

The number of elderly patients affected by gastric cancer has shown an increasing trend. Once diabetes is complicated by other diseases, body function becomes worse, which in turn increases the difficulty of treatment. Incidence of gastric cancer is correlated with diabetes, possibly due to living habits and family inheritance. The mechanism is complicated. Surgery is currently an effective method in the treatment of gastric cancer, but preoperative preparation, postoperative fasting, surgical trauma, anesthesia and other factors may affect blood glucose level, resulting in blood glucose fluctuations in patients, which can negatively affect postoperative rehabilitation, and the life and health of patients $(3,4)$. Therefore, monitoring of blood glucose is of great clinical significance for reducing the incidence of complications and promoting the prognosis of gastric cancer patients combined with T2MD.

A retrospective case-control study, which included elderly patients with gastric cancer that underwent radical operation, was conducted in this study to investigate changes in blood glucose and the effects of medication adjustment on rehabilitation and prognosis of those patients.

\section{Materials and methods}

General data. A total of 46 elderly patients with gastric cancer combined with T2DM who underwent radical operation from 
Table I. General data of study subjects.

\begin{tabular}{lcccc}
\hline & $\begin{array}{c}\text { DM group } \\
\mathrm{n}=46\end{array}$ & $\begin{array}{c}\text { Non-DM group } \\
\mathrm{n}=30\end{array}$ & $\mathrm{t} / \chi^{2}$ & $\mathrm{P}$-value \\
\hline Items & $65.4 \pm 9.5$ & $67.2 \pm 10.3$ & $\mathrm{t}=0.7826$ & $\mathrm{P}>0.05$ \\
Sex (years) & & & $\chi^{2}=0.7826$ & $\mathrm{P}>0.05$ \\
$\quad$ Male & 26 & 16 & \\
Female & 20 & 14 & $\mathrm{P}>0.05$ \\
Tumor location (n) & 16 & & $\chi^{2}=0.304$ & \\
Upper part of the cancer (U part) & 16 & 8 & \\
Medium part of the cancer (M part) & 14 & 8 & & \\
Lower part of the cancer (L part) & 26 & 14 & & \\
TNM staging & 20 & 14 & & \\
I & 0 & 0 & & \\
II & $246.2 \pm 159.4$ & $229.8 \pm 186.7$ & $\mathrm{t}=0.098$ & $\mathrm{P}>0.05$ \\
III & $210.4 \pm 96.5$ & $205.7 \pm 97.3$ & $\mathrm{t}=0.272$ & $\mathrm{P}>0.05$ \\
Intraoperative blood loss (ml) & $6.0 \pm 2.6$ & $5.2 \pm 2.1$ & $\mathrm{t}=1.473$ & $\mathrm{P}>0.05$ \\
Operation time (min) & & & \\
First defecation time (days) & & & \\
\hline
\end{tabular}

January 2007 to January 2017 and 30 gastric cancer patients without T2DM were included in the study. All the patients were subjected to total abdominal computed tomography (CT) and nuclear magnetic examination, and all of them were confirmed to be in line with the indications of radical operation for gastric cancer. In addition, all the patients received adjuvant chemotherapy. Patients combined with other malignant tumors other than gastric cancer, severe cardiovascular and cerebrovascular diseases, cachexia and inability for self-care were excluded. All enrolled patients were informed of the operation condition, and they or their family members signed the informed consent. This study was approved by the Ethics Committee of The First Hospital of Lanzhou University (Lanzhou, China). Patients were divided into the DM group and non-DM group according to the diagnosis of T2DM. T2DM diagnosis was in line with the diagnostic criteria of the World Health Organization (WHO) (5).

General clinical data of the 76 elderly patients, including age, sex, tumor location, tumor-node-metastasis (TNM) staging and pathological type were recorded and summarized. Pathological type was determined based on the 4th edition of 2010 WHO Classification of Tumors of the Digestive System (6). Gastric cancer staging was performed using the methods described in the 7th edition of 2010 Union for International Cancer Control (UICC) TNM Staging System of Gastric Cancer, and the tumor location was determined based on the 3rd edition of Japanese gastric cancer treatment guidelines $(7,8)$. The DM group comprised 26 males and 20 females, with an age range of 60-70 years and a mean age of $65.4 \pm 9.5$ years. The non-DM group comprised 16 males and 14 females, with an age range of 60-73 years and a mean age of $67.2 \pm 10.3$ years. Levels of preoperative blood glucose of the two groups of patients were controlled to reach the standard.
As shown in Table I, no significant differences in general data and preoperative blood glucose control were found between the two groups of patients $(\mathrm{P}>0.05)$. In 46 patients with gastric cancer combined with T2DM, 24 patients received adjustment of postoperative blood glucose with insulin, and 22 patients received no drug intervention to control blood glucose.

Methods. In 46 patients combined with DM, 24 patients were included in the insulin treatment group, and those patients were treated with continuous injection of insulin pumps to adjust blood glucose, and the initial injection dose was determined based on the patient's blood glucose level, age, body weight, and regimens in the early phase. Blood glucose was monitored 4 times a day, and the dose of insulin was adjusted according to the blood glucose level to avoid significant fluctuations in blood glucose, thus preventing the occurrence of hypoglycemia. The remaining 22 patients combined with T2DM were included in the non-insulin treatment group, and were treated with postoperative routine treatment, and insulin intervention was not performed.

According to condition of the clinical recovery after operation, postoperative blood glucose was controlled at 8.4-11.2 mmol/1, and blood glucose level was measured every $6 \mathrm{~h}$. Blood glucose level higher than this range in two measurements indicated poor blood glucose control. Fasting blood was extracted from each patient in the early morning. Serum carbohydrate antigen 19-9 (CA19-9) was detected using Roche Cobas E601 electrochemical luminescence spectrometer. Serum carcinoembryonic antigen (CEA) was determined by enzyme-linked immunosorbent assay (ELISA). Fasting elbow venous blood $(6 \mathrm{ml})$ was extracted from each patient in the early morning before and after treatment. Blood sample was stored in tubes containing ethylenediamine tetraacetic acid (EDTA), 
Table II. Changes in perioperative blood glucose of patients with gastric cancer in the DM group and the non-DM group (mmol/1, mean $\pm \mathrm{SD})$.

\begin{tabular}{lcccc}
\hline Items & DM group & Non-DM group & t value & P-value \\
\hline Case $(\mathrm{n})$ & 46 & 30 & & \\
Blood glucose before operation & $16.98 \pm 1.64$ & $4.01 \pm 1.75$ & 10.21 & 0.009 \\
Blood glucose at 24 h after operation & $24.09 \pm 1.42$ & $5.71 \pm 1.03$ & 8.12 & 0.02 \\
Blood glucose at 3 d after operation & $22.52 \pm 2.08$ & $5.09 \pm 1.22$ & 11.43 & 0.001 \\
\hline
\end{tabular}

and $3 \mathrm{ml}$ blood from each sample was used for white blood cell counting, while the remaining $3 \mathrm{ml}$ was used to prepare serum. White blood cells were counted using an automatic blood cell analyzer (XS800i, Sysmex Corporation, Kobe, Japan). Serum markers were detected by an automatic biochemical analyzer (Roche Modular P800, Roche Diagnostics GmbH, Mannheim, Germany) and immunoturbidimetric assay.

Follow-up. After the first postoperative chemotherapy, the patients began to receive long-term follow-up through telephone from the date of discharge to January 2017 or their death.

Statistical analysis. Statistical Product and Service Solutions (SPSS) 21.0 software was used for all statistical analyses. Kaplan-Meier method was used to calculate the 5-year survival rate. Measurement data were expressed as mean $\pm \mathrm{SD}$ and the t-test was used for comparisons between two groups. The $\chi^{2}$ test was used for comparisons of count data. Pairwise t-test was used for comparisons of data at two time-points within a group, and analysis of variance followed by post hoc test (Least Significant Difference) was used for comparisons among multiple time points within a group. $\mathrm{P}<0.05$ was considered to indicate a statistically significant difference.

\section{Results}

Changes in perioperative blood glucose in the DM and non-DM groups. Blood glucose levels in the DM group were significantly higher than those in the non-DM group before and at $24 \mathrm{~h}$ and 3 days after operation $(\mathrm{P}<0.05)$. Blood glucose concentrations after operation were significantly increased in DM group compared with the preoperative levels $(\mathrm{P}<0.05)$ (Table II).

Changes in postoperative blood glucose in the insulin treatment and non-insulin treatment groups. At 3 days after the treatment, concentration of blood glucose in the treatment group was significantly lower than that in non-treatment group $(\mathrm{P}<0.05)$. Blood glucose level of the non-treatment group was slowly recovered, and significant decreases were observed on the $3 \mathrm{rd}, 6 \mathrm{th}, 9 \mathrm{th}, 12$ th and 15 th day after operation. Decreases in level of blood glucose were more significant in insulin treatment group than in non-insulin treatment group (Fig. 1).

Changes in levels of gastric cancer markers in insulin treatment group and non-insulin treatment group before and after treatment. Levels of gastric markers, CEA and CA 19-9 in the treatment and non-treatment groups before and 3 months after operation were recorded and compared. Differences were not

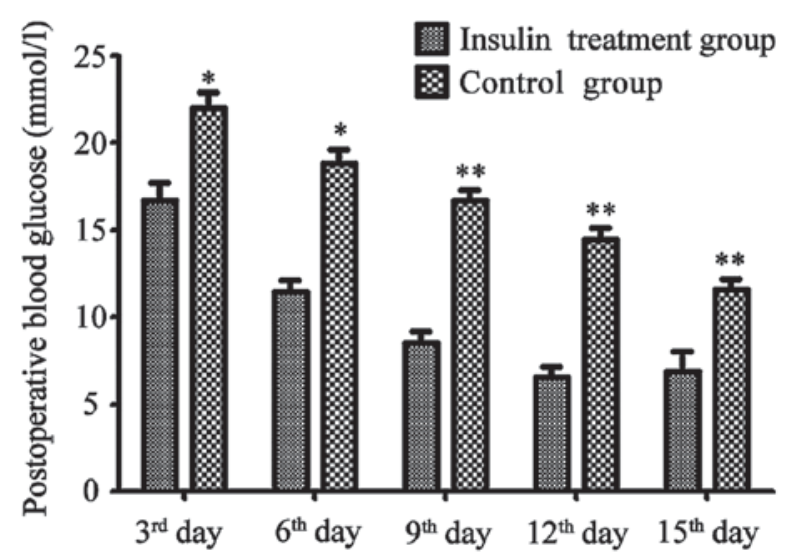

Figure 1. Changes in postoperative blood glucose of patients in insulin-treated group (24 cases) and non-insulin treated group (22 cases) before and after treatment. ${ }^{*} \mathrm{P}<0.01$ compared with insulin-treated group; ${ }^{* *} \mathrm{P}<0.05$ compared with insulin treated group.

significant $(\mathrm{P}>0.05)$. Compared with the preoperative levels, levels of carcinoembryonic antigen in both groups were significantly decreased after operation $(\mathrm{P}<0.05)$, and decreases were more significant in insulin treatment group than in control group $(\mathrm{P}<0.05)$ (Fig. 2A). Compared with preoperative levels, levels of carcinoembryonic antigen in both groups were significantly decreased after operation $(\mathrm{P}<0.05)$, and decreases were more significant in the insulin treatment group than in the control group $(\mathrm{P}<0.05)$ (Fig. 2B and Table III).

Changes in inflammatory response indexes, white blood cells and CRP in the insulin and non-insulin treatment groups. Changes in inflammatory response indexes, white blood cells and CRP in insulin treatment group and non-insulin treatment group on the 1st, 3rd and 5th day after operation were recorded. Results showed that levels of inflammatory response indexes in the two groups of patients on the 5th day after operation were significantly decreased compared with those on the 1st day after operation. Decreases in white blood cells were more significant in patients of the insulin treatment group than those in the control group $(\mathrm{P}<0.05$, Fig. 3). Decreases in CRP were more significant in patients of the insulin treatment group than those in the control group. $(\mathrm{P}<0.05$, Fig. 4$)$

Comparisons of postoperative hospital stay, poor postoperative blood glucose control, and poor incision healing between the insulin and non-insulin treatment groups. In the non-treatment group, hospital stay of elderly patients 
Table III. Levels of gastric cancer markers in the insulin treatment group and the non-insulin treatment group before and after treatment.

\begin{tabular}{lccrr}
\hline Items & $\begin{array}{c}\text { Insulin treatment } \\
\text { group }(\mathrm{n}=24)\end{array}$ & $\begin{array}{c}\text { Non-insulin treatment } \\
\text { group }(\mathrm{n}=22)\end{array}$ & t value & P-value \\
\hline Preoperative CEA $(\mu \mathrm{g} / \mathrm{l})$ & $17.4 \pm 6.5$ & $16.4 \pm 9.5$ & 2.032 & 0.541 \\
Postoperative CEA $(\mu \mathrm{g} / \mathrm{l})$ & $1.4 \pm 9.6^{\mathrm{a}}$ & $6.9 \pm 9.7^{\mathrm{a}}$ & 10.765 & 0.014 \\
Preoperative CA 19-9 $(\mathrm{kU} / \mathrm{l})$ & $210.4 \pm 96.5$ & $220.4 \pm 97.5$ & 2.986 & 0.492 \\
Postoperative CA 19-9 $(\mathrm{kU} / \mathrm{l})$ & $90.4 \pm 87.5^{\mathrm{a}}$ & $110.4 \pm 77.8^{\mathrm{a}}$ & 8.231 & 0.026 \\
\hline
\end{tabular}

${ }^{a} \mathrm{P}<0.05$ represents that compared with those before operation within the group.
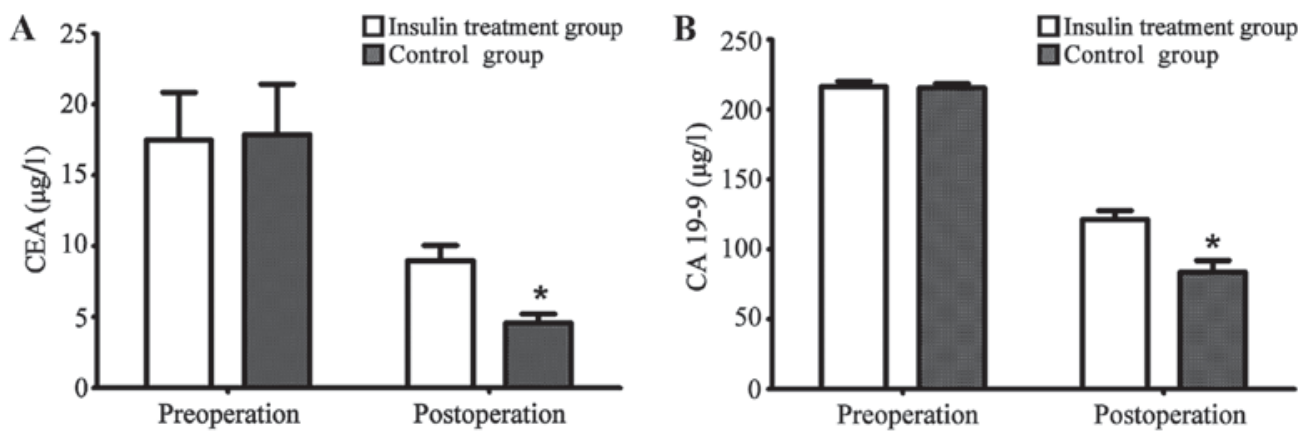

Figure 2. Levels of gastric cancer markers in the two groups before and after treatment $\left({ }^{*} \mathrm{P}<0.05\right)$. (A) Levels of carcinoembryonic antigen in the insulin treated group ( 24 cases) and non-insulin treated group ( 22 cases) both decreased. After treatment, the level of carcinoembryonic antigen in the treatment group was lower than that in non-treatment group. (B) Concentration of carbohydrate antigen 19-9 (CA19-9) decreased after operation in both treatment group (24 cases) and untreated group (22 cases). After treatment, level of CA19-9 in treatment group was lower than that in untreated group. ${ }^{*} \mathrm{P}<0.05$ compared with the control group.

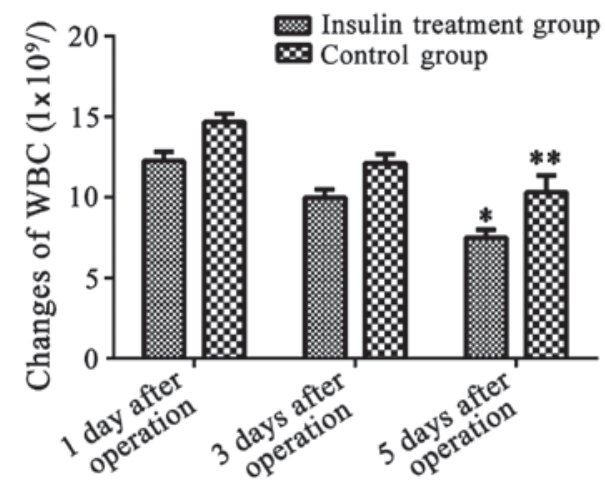

Figure 3. Changes of white blood cells in the two groups before and after treatment $\left({ }^{*} \mathrm{P}<0.05,{ }^{* *} \mathrm{P}<0.01\right)$. On day 5 , level of white blood cells in the treatment group ( 24 cases) was significantly lower than that in the untreated group (22 cases). ${ }^{*} \mathrm{P}<0.05$ compared with the control group; ${ }^{* *} \mathrm{P}<0.01$ compared to 1 day after operation.

was $12 \pm 2$ days, which was significantly longer than that in treatment group $(9 \pm 1$ days $)(\mathrm{P}<0.05)$. Incidence rate of poor incision healing was $12.5 \%$ in patients of the insulin treatment group, and $63.6 \%$ in patients of the non-treatment group. The incidence rate of poor incision healing in the non-insulin treatment group $(90.9 \%)$ was significantly higher than that in treatment group $(25.0 \%)$ (Table III).

Comparison of the 5-year survival rate of patients between insulin treatment group and non-insulin treatment group. The

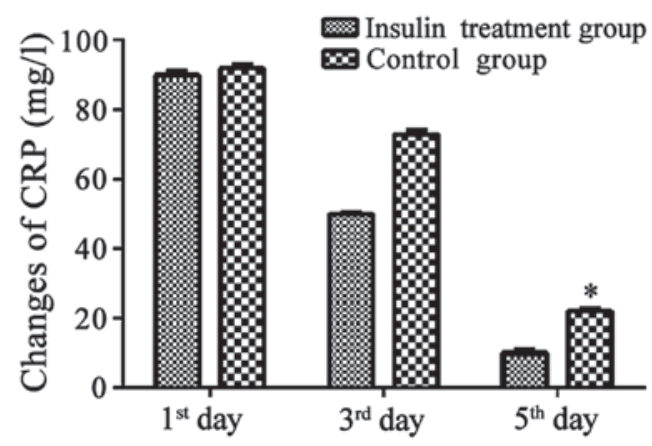

Figure 4. Changes of CRP in the two groups before and after treatment $\left({ }^{*} \mathrm{P}<0.05\right)$. On day 5, level of CRP in treatment group (24 cases) was significantly lower than that in the untreated group ( 22 cases). ${ }^{*} \mathrm{P}<0.05$ compared with the control group.

5-year survival rates in the insulin treatment group and the non-insulin treatment group were 66.67 and $40.90 \%$, respectively. Survival time of patients in the insulin treatment group was significantly longer than that of patients in non-insulin treatment group $(\mathrm{P}<0.05)$ (Fig. 5).

\section{Discussion}

Gastric cancer is one of the most common malignant gastrointestinal tumors with increasing incidence rate (9). Radical resection is the only effective mean for treating gastric cancer. With the development of surgical techniques, incidence rate of 
Table IV. Postoperative hospital stay and poor incision healing in the insulin treatment group and the non-insulin treatment group.

\begin{tabular}{lcccr}
\hline Groups & $\begin{array}{c}\text { Insulin treatment } \\
\text { group }(\mathrm{n}=24)\end{array}$ & $\begin{array}{c}\text { Non-insulin treatment } \\
\text { group }(\mathrm{n}=22)\end{array}$ & t value & P-value \\
\hline Hospital stay (days) & $9 \pm 1$ & $12 \pm 2$ & 5.56 & 0.021 \\
Poor incision healing [n (\%)] & $3(12.5 \%)$ & $14(63.6 \%)$ & 9.32 & 0.034 \\
Poor postoperative blood glucose control [n (\%)] & $6(25.0 \%)$ & $20(90.9 \%)$ & 5.16 & 0.020 \\
\hline
\end{tabular}

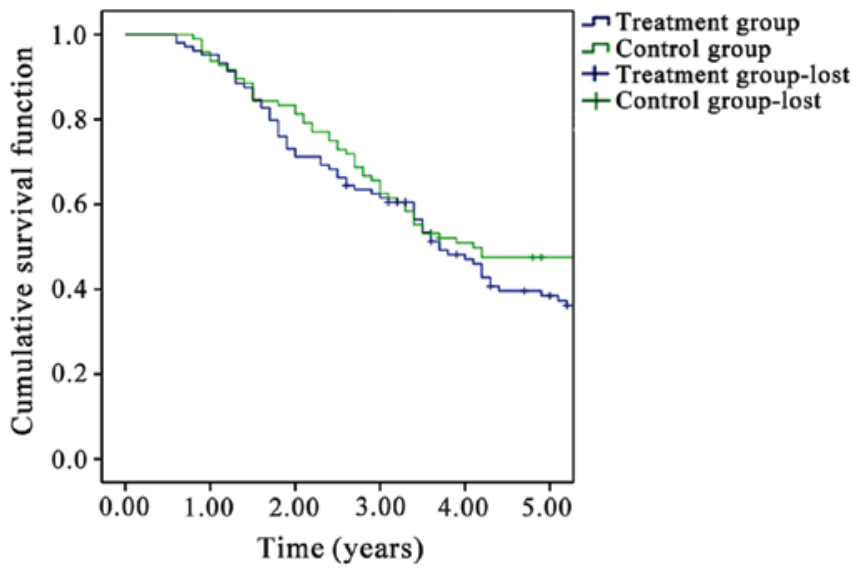

Figure 5. Comparison of the survival curve of elderly patients between the insulin treatment group and the non-insulin treatment group. The survival rate of patients in the treatment group is higher than that in the non-treatment group $(\mathrm{P}<0.05)$. Treatment group-lost refers to patients that died during the follow-up in the treatment group. Control group lost refers to patients that died during the follow-up in the control group.

postoperative complications of gastric cancer has been significantly reduced. However, surgery-related factors may increase the risk of infections, leading to postoperative death (10). At present, with an aging population that continues to grow, incidence rate of T2DM is increased year by year, and blood glucose level is an important factor affecting the recovery of gastric cancer after radical operation (11).

Incidence rate of poor postoperative blood glucose control in elderly patients of non-treatment group (90.9\%) was significantly higher than that in insulin treatment group (25\%), which was consistent with previous studies. At the same time, radical operation performed for DM patients can easily trigger or aggravate the complications of DM, and also can greatly increase the incidence of complications after gastrectomy. Mortality rate of surgery and incidence rate of complications are approximately 5 times higher in DM patients than in patients without DM (12). In addition, poor blood glucose control and surgical stimulations will trigger the stress responses and increase the level of blood glucose. Insulin can be secreted to resist the elevated blood glucose, while patients with islet dysfunction or a history of DM usually have poor hypoglycemic ability.

A study has shown that blood glucose controlling through perioperative drug intervention can significantly reduce the incidence rate of complications (10), which is consistent with our study findings. Comparison of the 5-year survival rate of elderly patients between insulin treatment group and non-insulin treatment group showed that survival time is significantly longer in insulin treatment group than that in non- insulin treatment group. At the same time, a meta-analysis showed that the combination of DM with malignant tumors could significantly increase the mortality rate of patients (13). Another recent meta-analysis in 2017 revealed that patients with T2DM treated with melbine have a lower risk of gastric cancer than those who were not treated with melbine (14). In addition, perioperative glycemic control can not only reduce the incidence rate of postoperative complications, but also can benefit patients in the long-term prognosis because early blood glucose control can delay the deterioration of tumor to some extent and improve the survival of patients $(15,16)$. These studies are consistent with our conclusions.

Elderly patients have special clinical characteristics, such as dormant clinical manifestations, more preoperative complications, poor postoperative tolerance and weak cardiovascular and respiratory system functions. Thus, early intervention and treatment are needed for those patients. DM and poor postoperative glycemic control are common causes of infection, and a study has shown that prolonged hospital stay may even cause death (17). Infection is mainly reflected by incision healing condition. Our results showed that the incidence rates of poor incision healing in insulin treatment group and the non-insulin treatment group were 12.5 and $63.6 \%$, respectively, and the difference was not statistically significant. It was believed that the inconsistency of the results might be related to the relatively small sample size. Among 46 elderly patients combined with T2DM underwent radical operation, incidence rate of poor incision healing in the drug intervention group was significantly lower than that in non-drug treatment group, which was likely to be related to stress hyperglycemia of the body.

Stress hyperglycemia may lead to immune dysfunction to promote the release of inflammatory factors, aggravate the systemic inflammatory responses and increase the incidence of infection, thus leading to a series of complications (18). Drug intervention in blood glucose control can not only improve the utilization of glucose in the body, but also can enhance the metabolism, reduce catabolism, and indirectly reduce the incidence rate of inflammatory responses. Therefore, blood glucose control with drug intervention can significantly promote the rehabilitation of patients and reduce the occurrence of complications (19). A comparison of prognosis between the elderly patients and non-elderly patients after radical operation has shown that, although elderly patients with gastric cancer have relatively more preoperative complications, strengthened perioperative treatment could significantly reduce incidence rate of complications and mortality rate to reach normal level (20). 
Therefore, postoperative blood glucose control through drug intervention is of great clinical significance for improvement of treatment outcomes, especially for elderly patients.

In this study, elderly patients were selected as subjects, and the changes in blood glucose of these elderly patients with gastric cancer combined with T2DM after radical operation were investigated, and the effects of medication adjustment on the recovery and prognosis of gastric cancer were explored. Our study provided reference for the treatment of gastric cancer combined with T2DM. It was believed that DM might be an independent risk factor for poor prognosis of gastric cancer patient underwent radical operation. For the elderly patients combined with DM, comprehensive preoperative assessment and positive postoperative adjustment of blood glucose can significantly reduce incidence of postoperative complications and mortality rate, thereby enhancing the long-term survival rate.

\section{Acknowledgements}

Not applicable.

\section{Funding}

This study was supported by Natural Science Foundation of Gansu Province (1308RJZA219).

\section{Availability of data and materials}

All data generated or analyzed during this study are included in this published article.

\section{Authors' contributions}

YZ and SL designed the study. JW and XY collected the patient data. LZ and YZ analyzed the patient data. All authors have read and approved the final manuscript.

\section{Ethics approval and consent to participate}

All enrolled patients were informed of the operation condition, and they or their family members signed the informed consent. This study was approved by the Ethics Committee of The First Hospital of Lanzhou University (Lanzhou, China).

\section{Patient consent for publication}

Not applicable.

\section{Competing interests}

The authors declare no competing interests.

\section{References}

1. Ferlay J, Soerjomataram I, Dikshit R, Eser S, Mathers C, Rebelo M, Parkin DM, Forman D and Bray F: Cancer incidence and mortality worldwide: Sources, methods and major patterns in GLOBOCAN 2012. Int J Cancer 136: E359-E386, 2015.
2. Chang-Chen KJ, Mullur R and Bernal-Mizrachi E: Beta-cell failure as a complication of diabetes. Rev Endocr Metab Disord 9: 329-343, 2008.

3. Chan RP, Galas FR, Hajjar LA, Bello CN, Piccioni MA and Auler JO Jr: Intensive perioperative glucose control does not improve outcomes of patients submitted to open-heart surgery: A randomized controlled trial. Clinics (Sao Paulo) 64: 51-60, 2009.

4. Lavernia F: Treating hyperglycemia and diabetes with insulin therapy: Transition from inpatient to outpatient care. Medscape J Med 10: 216, quiz 216, 2008.

5. Rongli Q: The significance of new diagnostic criteria and typing of diabetes mellitus. J Clin Int Med 17: 133, 2000.

6. Teng XD and Lai DM: New progress of gastric pathology - 2010 WHO Classification of Tumors of the Digestive System. Chin J Clin Exp Pathol 28: 121-123, 2012.

7. Kwon SJ: Evaluation of the 7th UICC TNM staging system of gastric cancer. J Gastric Cancer 11: 78-85, 2011.

8. Japanese Gastric Cancer Association: Japanese gastric cancer treatment guidelines 2010 (ver. 3). Gastric Cancer 14: 113-123, 2011.

9. Forman D and Burley VJ: Gastric cancer: Global pattern of the disease and an overview of environmental risk factors. Best Pract Res Clin Gastroenterol 20: 633-649, 2006.

10. Sugisawa N, Tokunaga M, Tanizawa Y, Bando E, Kawamura T and Terashima M: Intra-abdominal infectious complications following gastrectomy in patients with excessive visceral fat. Gastric Cancer 15: 206-212, 2012.

11. Dronge AS, Perkal MF, Kancir S, Concato J, Aslan M and Rosenthal RA: Long-term glycemic control and postoperative infectious complications. Arch Surg 141: 375-380, 2006.

12. Jacober SJ and Sowers JR: An update on perioperative management of diabetes. Arch Intern Med 159: 2405-2411, 1999.

13. Barone BB, Yeh HC, Snyder CF, Peairs KS, Stein KB, Derr RL, Wolff AC and Brancati FL: Long-term all-cause mortality in cancer patients with preexisting diabetes mellitus: A systematic review and meta-analysis. JAMA 300: 2754-2764, 2008.

14. Zhou XL, Xue WH, Ding XF, Li LF, Dou MM, Zhang WJ, Lv Z, Fan ZR, Zhao J and Wang LX: Association between metformin and the risk of gastric cancer in patients with type 2 diabetes mellitus: A meta-analysis of cohort studies. Oncotarget 8: 55622-55631, 2017

15. Pettit S, Cresta E, Winkley K, Purssell E and Armes J: Glycaemic control in people with type 2 diabetes mellitus during and after cancer treatment: A systematic review and meta-analysis. PLoS One 12: e0176941, 2017.

16. Miao ZF, Xu H, Xu YY, Wang ZN, Zhao TT, Song YX and $\mathrm{Xu}$ HM: Diabetes mellitus and the risk of gastric cancer: A metaanalysis of cohort studies. Oncotarget 8: 44881-44892, 2017.

17. Sah BK, Zhu ZG, Chen MM, Yan M, Yin HR and Zhen LY: Gastric cancer surgery and its hazards: Post operative infection is the most important complication. Hepatogastroenterology 55: 2259-2263, 2008

18. Orsenigo E, Tomajer V, Palo SD, Carlucci M, Vignali A, Tamburini A and Staudacher C: Impact of age on postoperative outcomes in 1118 gastric cancer patients undergoing surgical treatment. Gastric Cancer 10: 39-44, 2007.

19. Bagry HS, Raghavendran S and Carli F: Metabolic syndrome and insulin resistance: Perioperative considerations. Anesthesiology 108: 506-523, 2008.

20. Egi M and Bellomo R: Reducing glycemic variability in intensive care unit patients: A new therapeutic target? J Diabetes Sci Technol 3: 1302-1308, 2009.

This work is licensed under a Creative Commons Attribution-NonCommercial-NoDerivatives 4.0 International (CC BY-NC-ND 4.0) License. 